\title{
Coral Keratitis: Case Report and Review of Mechanisms of Action, Clinical Management and Prognosis of Ocular Exposure to Palytoxin
}

This article was published in the following Dove Press journal: Clinical Ophthalmology

\author{
Ryan T Barrett' \\ Jordan P Hastings ${ }^{2}$ \\ Yasmyne C Ronquillo (iD) ${ }^{3}$ \\ Phillip C Hoopes (D) $^{3}$ \\ Majid Moshirfar (1D ${ }^{3-5}$ \\ 'Hollingshead Eye Center, Boise, ID, \\ USA; ${ }^{2}$ California Northstate University \\ College of Medicine, Elk Grove, CA, \\ USA; ${ }^{3}$ Hoopes Vision Research Center \\ Draper, UT, USA; ${ }^{4}$ John A. Moran Eye \\ Center, University of Utah School of \\ Medicine, Salt Lake City, UT, USA; ${ }^{5}$ Utah \\ Lions Eye Bank, Murray, UT, USA
}

Correspondence: Majid Moshirfar HDR Research Center, Hoopes Vision, I 1820 S. State Street Suite \#200, Draper, UT, USA

Tel + I 80I-568-0200

Email Cornea2020@me.com

\begin{abstract}
Palytoxin is one of the most lethal natural toxins ever discovered. This molecule has been isolated from various marine animals, including zoanthid corals. This popular organism is commonly found in many home saltwater aquariums due to its beauty and survivability. As a result of an increase in popularity, an increased number of individuals are at risk for exposure to this potentially deadly toxin. Affected patients may experience various symptoms based on the route of exposure (ie, cutaneous contact, inhalation of aerosolized toxin, ocular exposure, or ingestion). Ocular exposure can occur in various ways (eg, contact with contaminated water, rubbing the eye with a dirtied hand, or direct spraying into the eye), and incidence rates have dramatically risen in recent years. In this review, we discuss a case of systemic toxicity from inhalation and ocular exposure to presumed palytoxin on a zoanthid coral which resulted in an intensive care unit (ICU) stay, and corneal perforation which required a corneal transplant. Additionally, we review what is known about the mechanism of action of this toxin, propose a comprehensive hypothesis of its effects on corneal cells, and discuss the prognosis and clinical management of patients with systemic symptoms secondary to other routes of exposure.
\end{abstract}

Keywords: coral keratitis, zoanthid, palytoxin, corneal perforation, case report

\section{Introduction}

Ancient Hawaiian legend tells of a half-man half-shark god who would prey on local fishermen. When the villagers found out the cause of the disappearing persons, they confronted, dismembered, and burned the god. When all was finished, they spread his cursed ashes in a nearby tidepool. "Limu-make-o-Hana" or seaweed of death from Hana began to grow in the pool, and warriors would dip their spears in the seaweed (which turned out to be coral) to make them lethal. ${ }^{1}$

The pool, its associated coral, and resultant toxin were discovered in $1971^{1}$ and synthesized in $1994 .^{2}$ The toxin was named palytoxin (PTX) and much has been learned about its toxic potential and mechanism of action in recent years. PTX is a large (2680 kilodaltons) nonprotein naturally occurring toxin that is produced by various marine animals such as zoanthid soft corals and microalgae. ${ }^{3}$ It is one of the most lethal natural toxins ever discovered, with a median lethal dose $\left(\mathrm{LD}_{50}\right)$ of $150 \mathrm{ng} / \mathrm{kg}$ body weight IV in mice. $^{4}$

Zoanthids are popular corals in home aquariums due to their beauty and robust nature, making them relatively easy to maintain. While some species within the 
genus carry the toxin, others are toxin-free. Since these corals are traded and sold without distinction as to their origin, identifying the presence of toxin based on the specific coral is not possible without genetic testing, and all zoanthid corals should be presumed to have toxic potential. ${ }^{5,6}$ Human exposure to the toxin is rare but has occurred with increased frequency within the last decade. Exposure can occur through direct contact with the skin or eyes, inhalation, or ingestion. ${ }^{7-10}$

In this paper, we report a case of palytoxin keratitis leading to corneal perforation. Additionally, we offer a review of the literature covering all published cases of ocular palytoxin exposure and discuss the mechanism of action of the toxin and prognosis after exposure. Finally, we discuss other routes of exposure and provide an overview of symptomatology and prognosis of patients who are exposed to palytoxin. This is the fourth reported case of corneal perforation secondary to palytoxin exposure and provides insight into the clinical course and surgical management of a patient with this toxicity that is increasing in frequency. ${ }^{11}$

A review of literature was done by searching the term palytoxin singularly, or in combination with the following words or phrases: "coral keratitis", "coral conjunctivitis", "soft coral", “zoanthid”, “cytoskeleton", “exposure”, "mechanism of action”, "poisoning”, "toxicity”, “ophthalmic", "ophthalmology", "inflammation", “eye”, “aquarium", and "case report" in PubMed and Google Scholar. The earliest article was published in 1993, with the most recent reports from 2020. Additional search engines were queried but revealed no additional cases. Searches were done with the terms "coral keratitis" and "coral conjunctivitis" and the other preceding terms. Eighteen published case reports were identified in eleven articles, and the results of these cases are summarized in Table 1. Finally, PubMed searches were done to explore and synthesize the effects of palytoxin via inhalational, gastrointestinal, and cutaneous routes of exposure.

\section{Case Report}

A 37-year-old Hispanic male with a past medical history significant for testicular cancer and nephrolithiasis and unremarkable past ocular history was examined in the hospital by the emergency department provider for right eye pain. The previous night, the patient was handling some coral he had bought online and cut his hand on the rock. Additionally, upon putting the coral in his aquarium, a plume of dust was released into the air. Within a couple of hours, he noticed severe right eye (OD) pain. At no point during his care did the patient have any complaints or abnormal findings on his left eye (OS). It is possible that he may have inadvertently touched the affected eye (OD) with his fingers but did not recall this when he gave his history.

The patient presented to the emergency room the morning after exposure where he was examined by an emergency room provider, given erythromycin ointment and discharged home with outpatient ophthalmology followup scheduled. Over the course of that day, his previously healthy dog got sick and died, and his cat developed respiratory symptoms. The patient's 17-year-old daughter and her boyfriend also developed a cough. The patient developed worsening eye pain and respiratory symptoms and re-presented to the ED that same night where he was evaluated by the ED provider, prescribed topical ciprofloxacin, and sent home again. The patient awoke the next morning ( 2 days after exposure) with eye pain and discharge, difficulty breathing, epigastric abdominal pain, streaks up his forearm, and diffuse pruritis. He represented a third time to the ED, was admitted to the hospital, and was later transferred to the ICU for increasing shortness of breath and a likely diagnosis of palytoxin exposure.

Chest and hand X-rays showed bibasilar infiltrates but no obvious signs of infection and a head CT (obtained over concerns for preseptal cellulitis) showed only preseptal tissue swelling. White blood count and C-reactive protein were elevated (18.3 and 200, respectively, on admission). Broad-spectrum antibiotics including meropenem $1000 \mathrm{mg}$ three times a day, linezolid $600 \mathrm{mg}$ two times a day, and vancomycin $15 \mathrm{mg} / \mathrm{kg}$ every eight hours were all started IV by the ICU team due to his symptoms and respiratory distress, and ophthalmology was consulted. Exam revealed visual acuity in the right eye was counting fingers, and he had 1+ edema of the upper and lower lids, chemosis, 2+ conjunctival injection, diffuse superior and peripheral punctate keratitis with a $360^{\circ}$ edematous cornea at the limbus. The pupil was miotic, presumed to be secondary to pain medication. The patient was diagnosed with presumed toxic palytoxin keratitis.

The patient was started on Tobradex (Alcon Laboratories, Inc) four times a day, cyclopentolate $2 \%$ two times per day, and artificial tears every two hours while awake. Fourteen hours later, his vital signs stabilized and his breathing had improved. Mucus was noted in the inferior fornix. A peripheral inflammatory ring and $1+$ folds in 


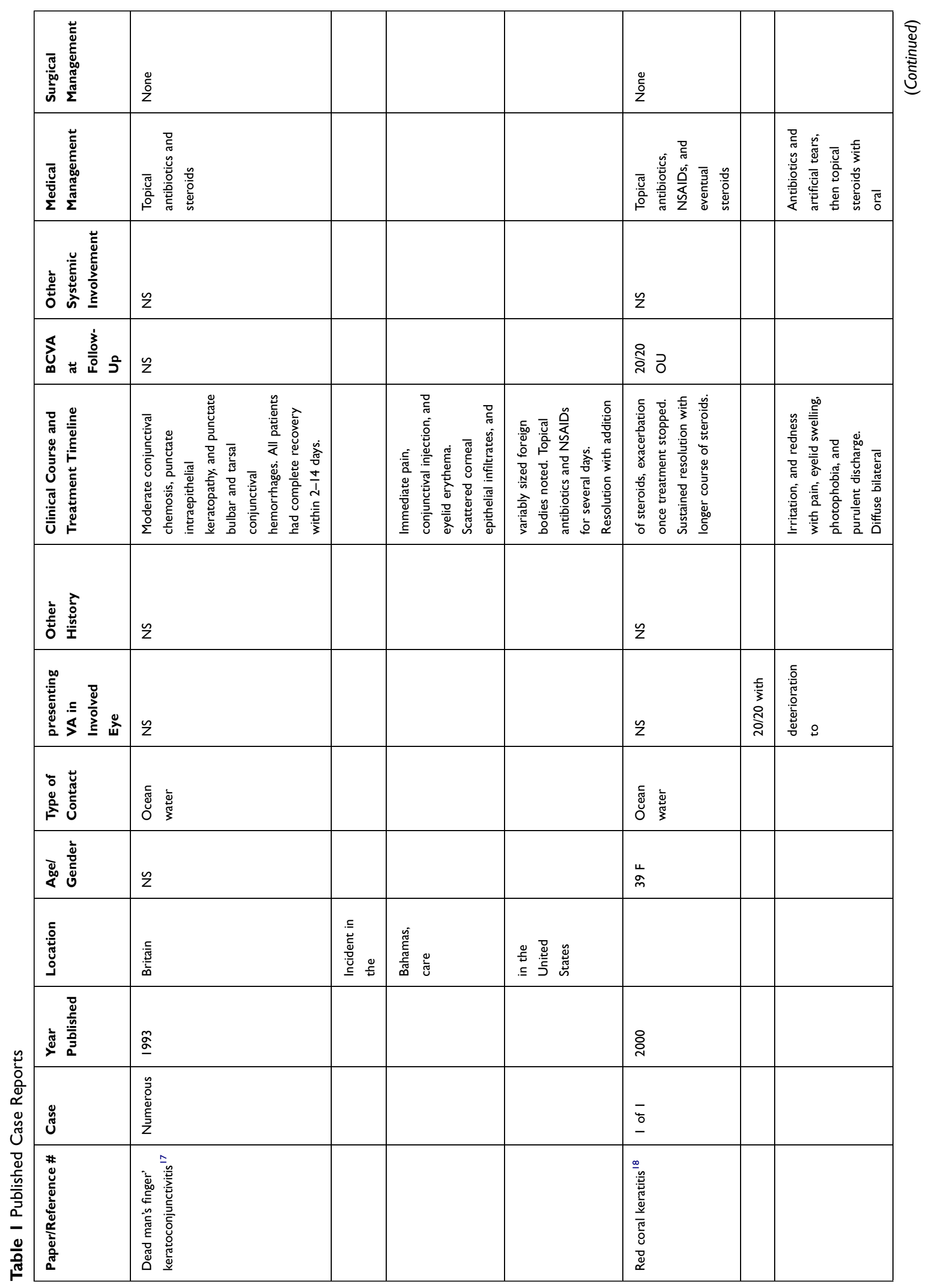




\begin{tabular}{|c|c|c|c|c|c|c|c|}
\hline 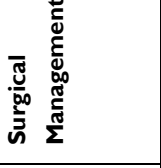 & & & $\begin{array}{l}\frac{0}{5} \\
\text { ż }\end{array}$ & $\begin{array}{l}\frac{0}{0} \\
\text { ż }\end{array}$ & & & 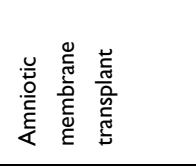 \\
\hline 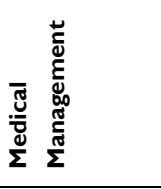 & 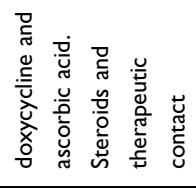 & 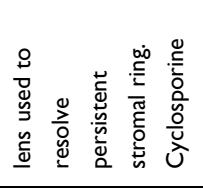 & 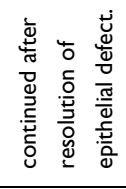 & 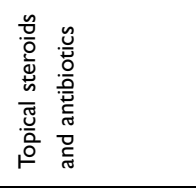 & & & \\
\hline 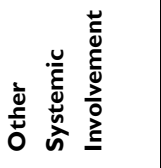 & & & 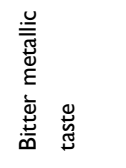 & $\tilde{z}$ & & 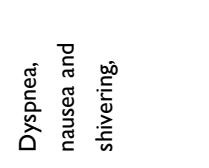 & 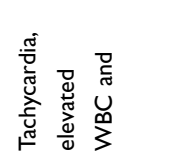 \\
\hline 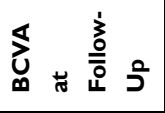 & & 突 & ő & ঙั઼ & & & 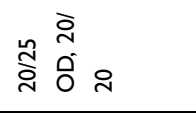 \\
\hline 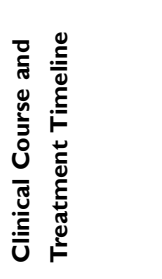 & 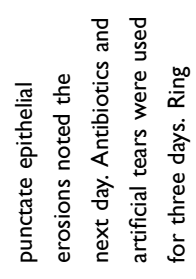 & 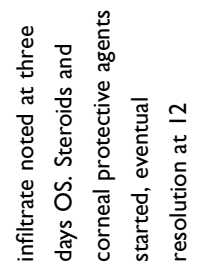 & 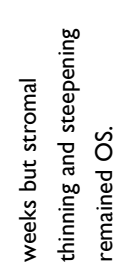 & 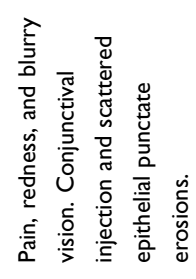 & & 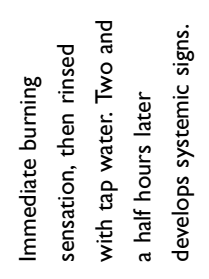 & 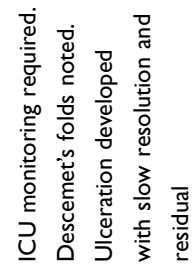 \\
\hline 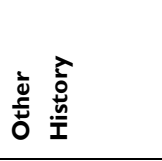 & & & 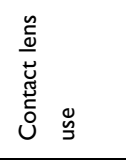 & $\begin{array}{l}\text { D } \\
\frac{1}{\bar{v}} \\
\text { s }\end{array}$ & & & \\
\hline 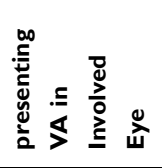 & 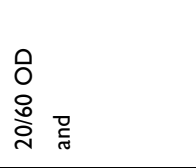 & 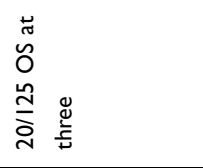 & 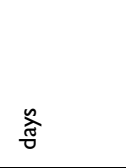 & 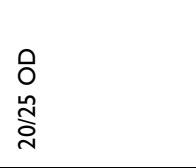 & & & \\
\hline 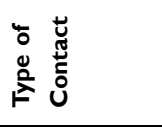 & & & $\tilde{z}$ & 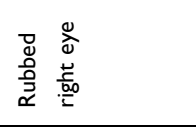 & & & \\
\hline 离 & & & $\frac{\Sigma}{m}$ & $\begin{array}{l}\sum \\
\underset{\sigma}{q}\end{array}$ & & & \\
\hline & & & 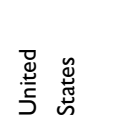 & 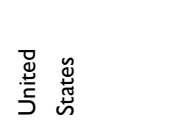 & & & \\
\hline 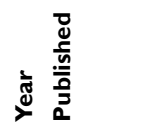 & & & $\frac{\circ}{\bar{N}}$ & $\frac{\circ}{\circ}$ & & & \\
\hline נֶ & & & $\frac{N}{0}$ & 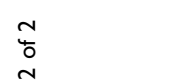 & & & \\
\hline 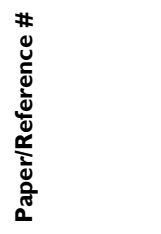 & & 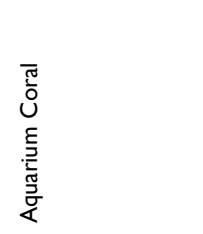 & 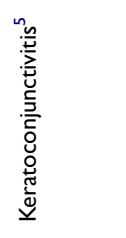 & 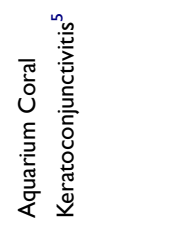 & 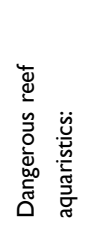 & 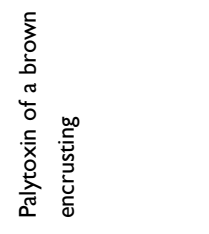 & 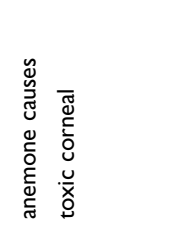 \\
\hline
\end{tabular}




\begin{tabular}{|c|c|c|c|}
\hline ठ & $\frac{0}{\frac{0}{2}}$ & $\frac{0}{\frac{0}{2}}$ & 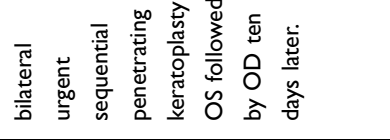 \\
\hline 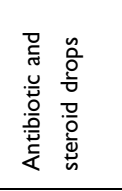 & 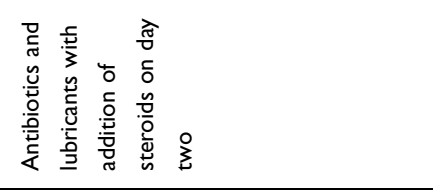 & 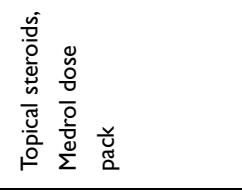 & 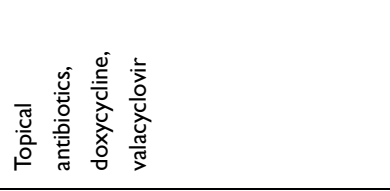 \\
\hline 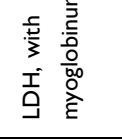 & $\tilde{z}$ & $\underline{n}$ & $\tilde{z}$ \\
\hline ๕̆ & $\underline{z}$ & ఫે̀ ว & 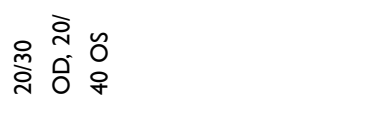 \\
\hline 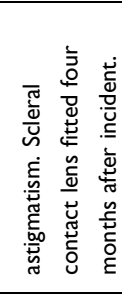 & 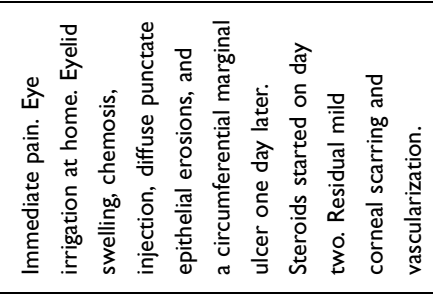 & 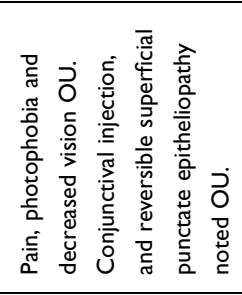 & 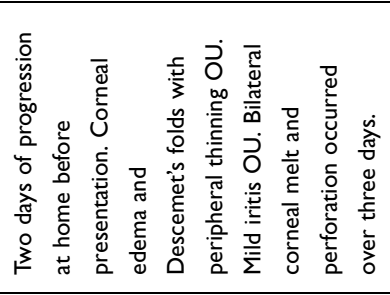 \\
\hline$\underline{z}$ & $\underline{z}$ & $\frac{\circ}{\frac{0}{2}}$ & $\begin{array}{l}8 \\
0 \\
\text { bे }\end{array}$ \\
\hline 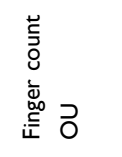 & 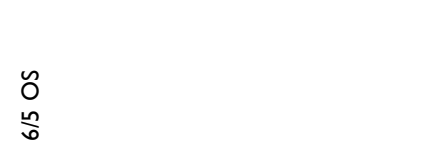 & 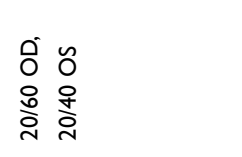 & 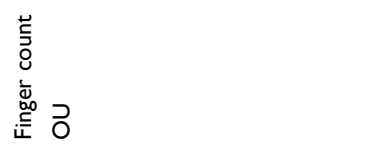 \\
\hline 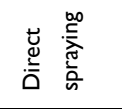 & 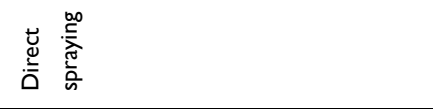 & $\underline{z}$ & $\check{z}$ \\
\hline 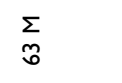 & $\stackrel{4}{\dot{y}}$ & 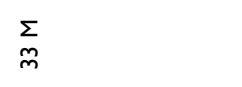 & $\Sigma$ \\
\hline 要 & 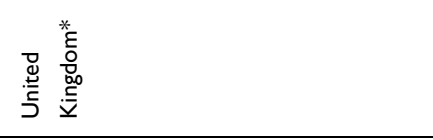 & 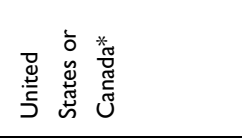 & 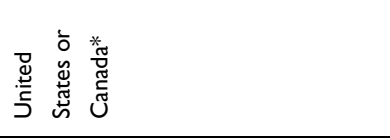 \\
\hline$\stackrel{n}{a}$ & $\stackrel{\circ}{\stackrel{\circ}{\circ}}$ & సे & $\overline{\bar{i}}$ \\
\hline$\overline{\bar{o}}$ & $\stackrel{\bar{o}}{-}$ & $\stackrel{1}{0}$ & $\stackrel{\grave{o}}{i}$ \\
\hline & 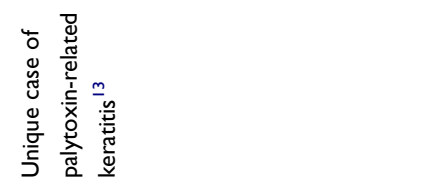 & 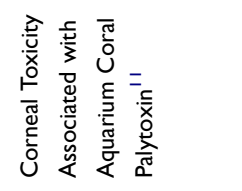 & 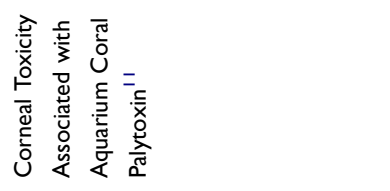 \\
\hline
\end{tabular}




\begin{tabular}{|c|c|c|c|}
\hline 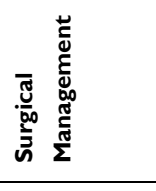 & $\begin{array}{l}\frac{0}{5} \\
\text { L } \\
\end{array}$ & 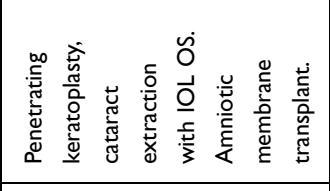 & 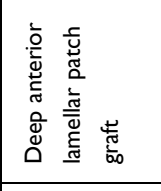 \\
\hline 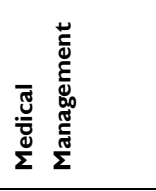 & 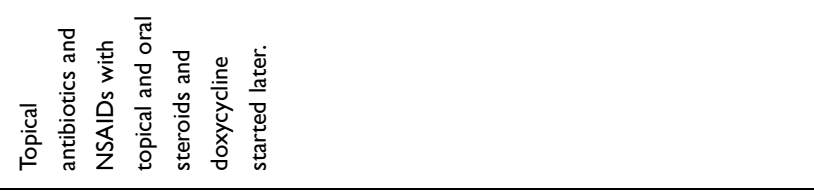 & 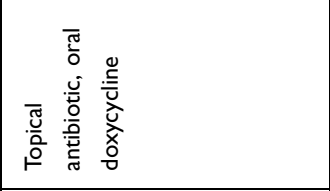 & $\tilde{z}$ \\
\hline 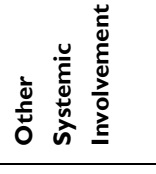 & $\tilde{z}$ & $\tilde{z}$ & $\tilde{z}$ \\
\hline 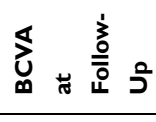 & ڤัํํ & 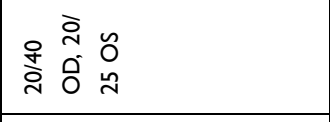 & 号 。 \\
\hline 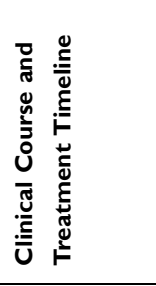 & 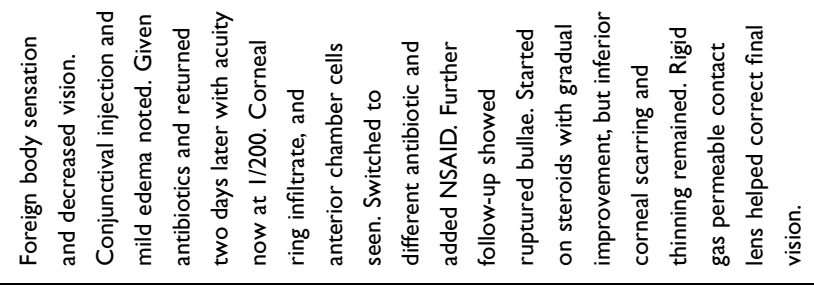 & 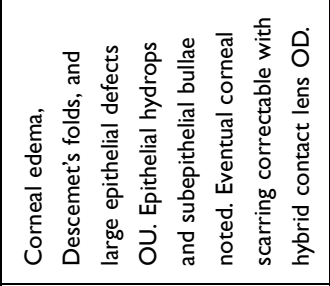 & 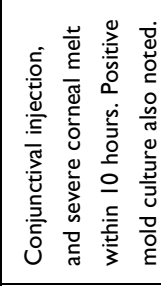 \\
\hline 竞高 & 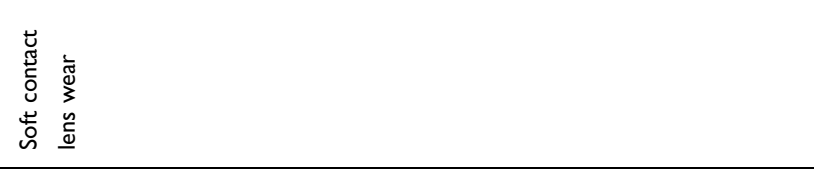 & $\underline{\underline{z}}$ & 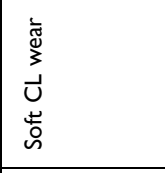 \\
\hline 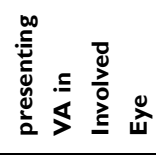 & $\begin{array}{l}\text { ô } \\
\text { ò } \\
\text { aें }\end{array}$ & \begin{tabular}{|l}
3 \\
0 \\
0 \\
0 \\
9 \\
\\
\end{tabular} & 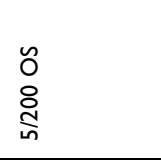 \\
\hline 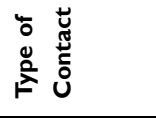 & $\underline{z}$ & $\underline{z}$ & $\underline{n}$ \\
\hline 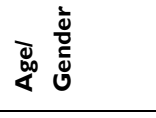 & 惢 & $\frac{\Sigma}{r}$ & $\underset{\neg}{\Sigma}$ \\
\hline 总 & 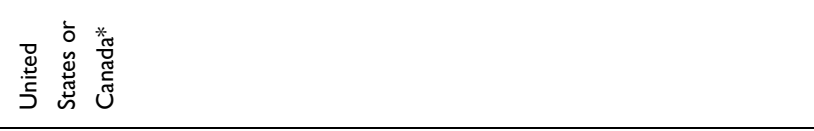 & 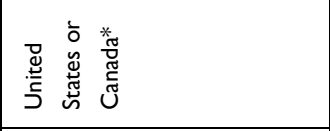 & 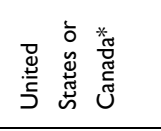 \\
\hline خ & $\bar{i}$ & iे & ì \\
\hline ֶّ & $\begin{array}{l}\hat{0} \\
\text { m }\end{array}$ & $\begin{array}{c}\frac{1}{0} \\
+\end{array}$ & $\begin{array}{l}\hat{\vdots} \\
\text { in }\end{array}$ \\
\hline 蒂 & 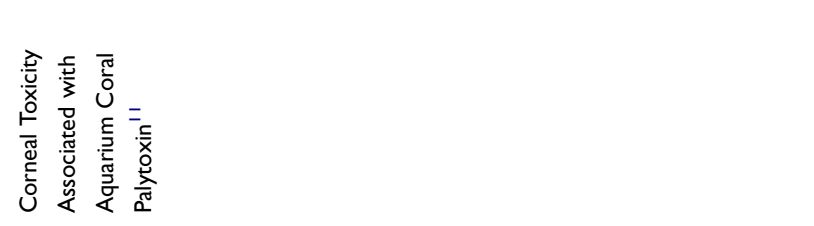 & 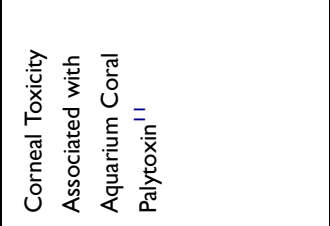 & 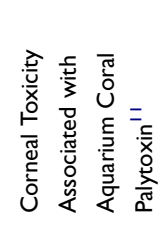 \\
\hline
\end{tabular}




\begin{tabular}{|c|c|c|c|c|c|}
\hline 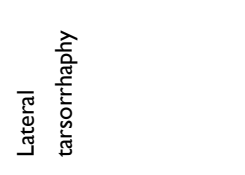 & 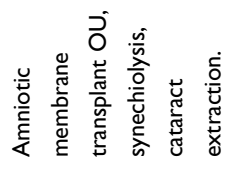 & & & & $\begin{array}{l}\stackrel{0}{0} \\
\text { zo }\end{array}$ \\
\hline 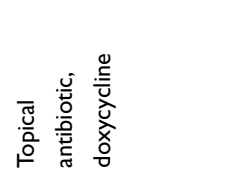 & 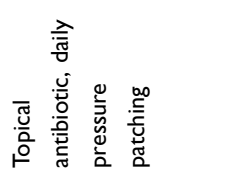 & & 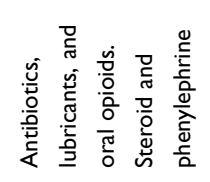 & 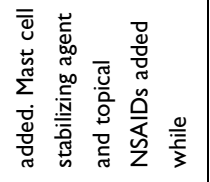 & 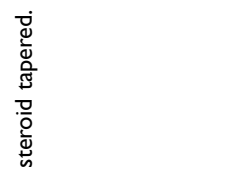 \\
\hline$\tilde{z}$ & $\tilde{z}$ & & & & $\tilde{z}$ \\
\hline 웜 ô & 高 o & & & & $\underset{\wedge}{\stackrel{N}{\grave{~}}}$ \\
\hline 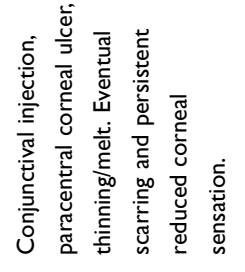 & 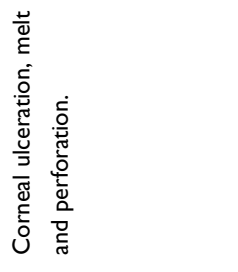 & & & 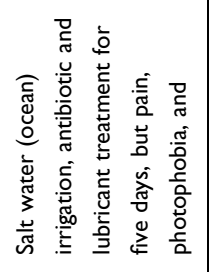 & 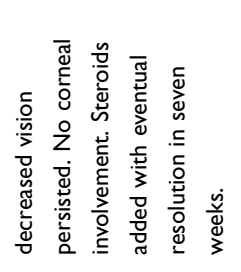 \\
\hline$\tilde{z}$ & $\tilde{z}$ & & & & 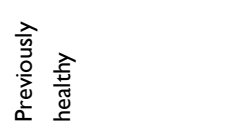 \\
\hline ฉ̊̊ & 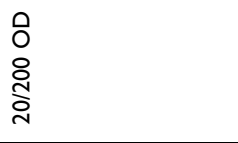 & & & & ণ্⿳亠丷冖巾 \\
\hline$\tilde{z}$ & $\tilde{z}$ & & & & 苛 \\
\hline $\begin{array}{l}\Sigma \\
\text { in }\end{array}$ & $\begin{array}{l}\Sigma \\
\text { \& }\end{array}$ & & & & 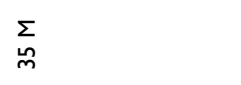 \\
\hline 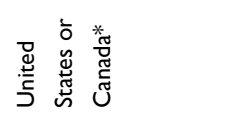 & 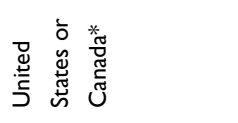 & & & & 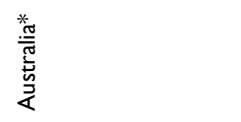 \\
\hline 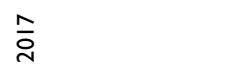 & $\overline{\bar{i}}$ & & & & $\overline{\bar{i}}$ \\
\hline $\begin{array}{l}\bar{y} \\
\bar{o} \\
0\end{array}$ & $\frac{1}{\grave{o}}$ & & & & 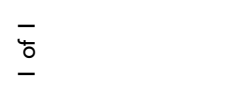 \\
\hline 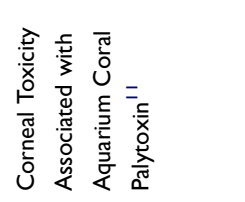 & 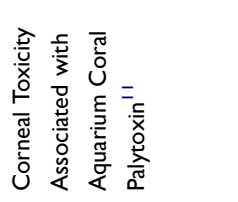 & 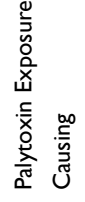 & 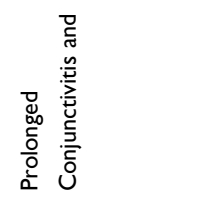 & 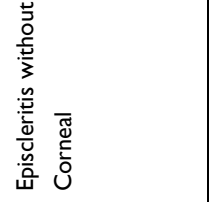 & 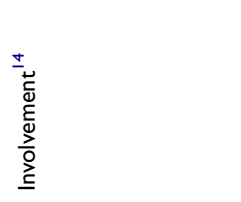 \\
\hline
\end{tabular}




\begin{tabular}{|c|c|c|}
\hline 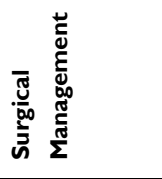 & 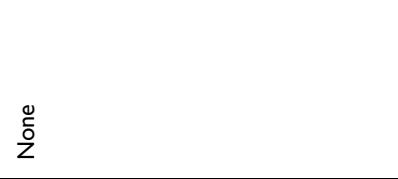 & 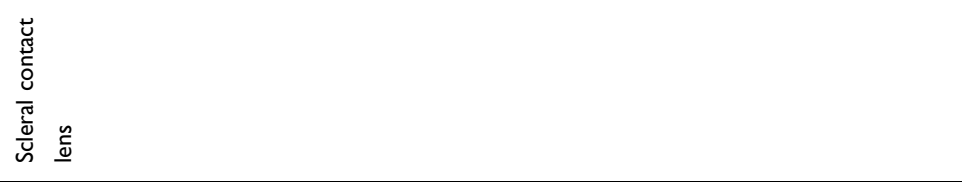 \\
\hline 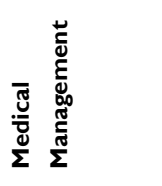 & 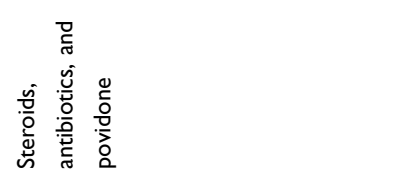 & 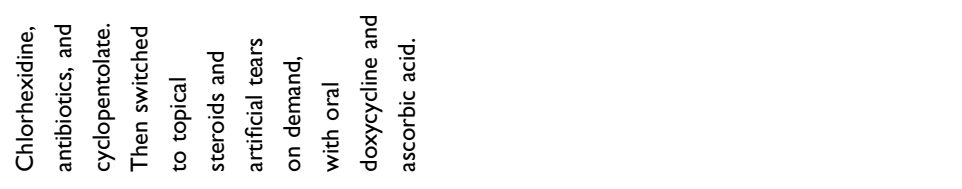 \\
\hline 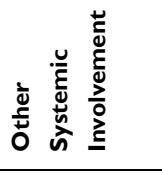 & 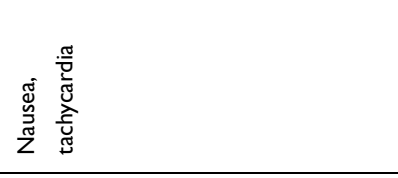 & $\tilde{z}$ \\
\hline ذِّ & 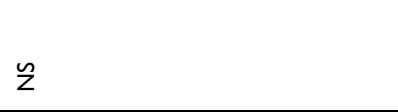 & 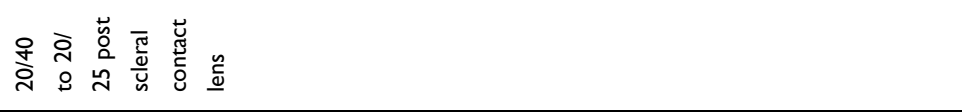 \\
\hline 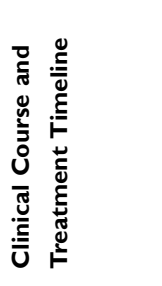 & 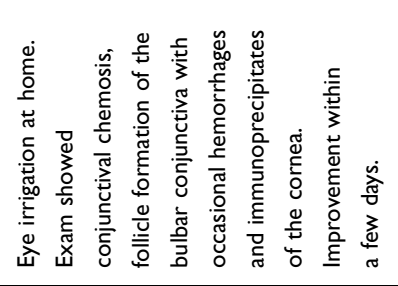 & 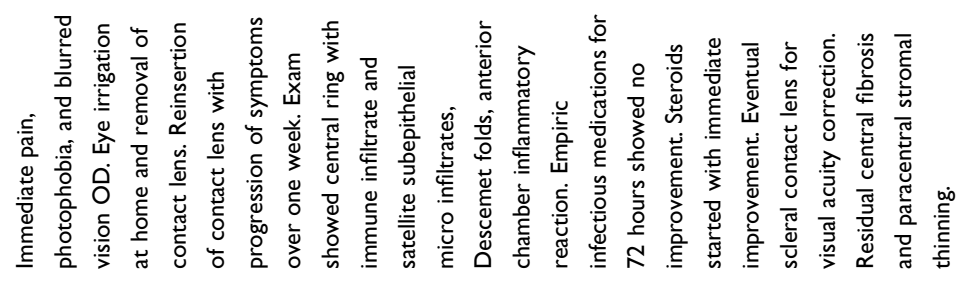 \\
\hline 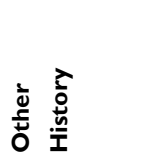 & 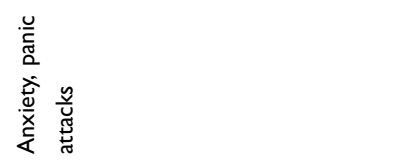 & 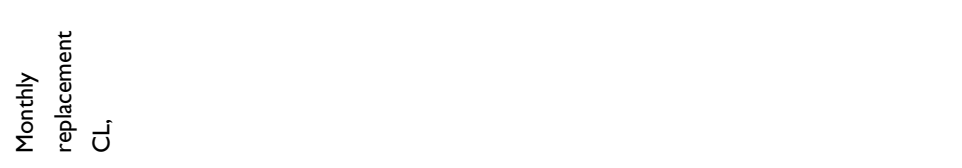 \\
\hline 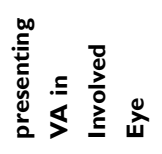 & $\stackrel{\sim}{\stackrel{\sim}{\sim}}$ & $\begin{array}{l}0 \\
0 \\
\circ \\
\stackrel{\circ}{\mathrm{O}} \\
\text { v }\end{array}$ \\
\hline 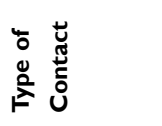 & 苛 & 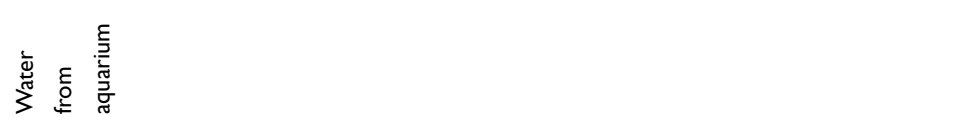 \\
\hline ঠ் & $\underset{\substack{\infty \\
m}}{\sum}$ & $\underset{⿱ 亠 乂}{\Sigma}$ \\
\hline د.ّ & 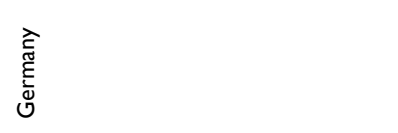 & 䞤 \\
\hline 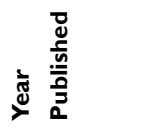 & $\frac{\circ}{\bar{N}}$ & $\stackrel{\circ}{\bar{\sim}}$ \\
\hline ঠ் & $\overline{\bar{o}}$ & $\overline{\bar{o}}$ \\
\hline 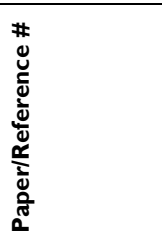 & 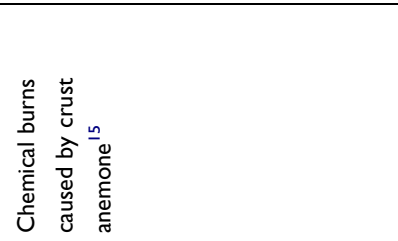 & 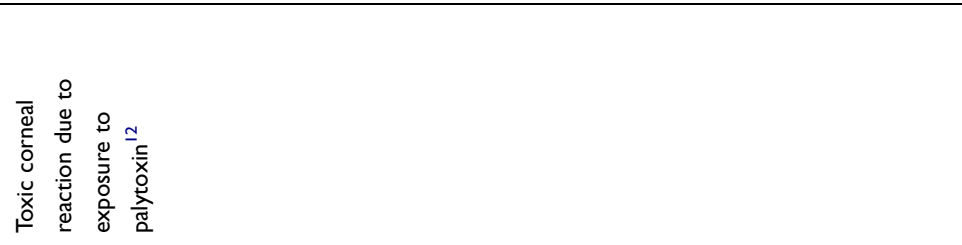 \\
\hline
\end{tabular}




\begin{tabular}{|c|c|}
\hline 总 & 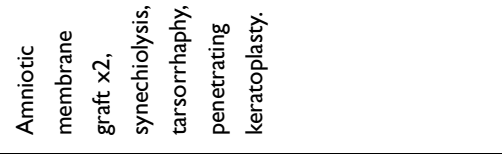 \\
\hline 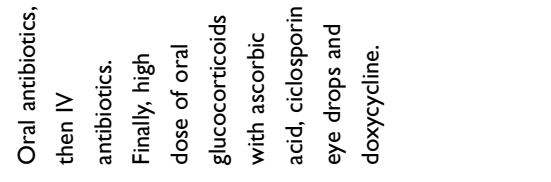 & 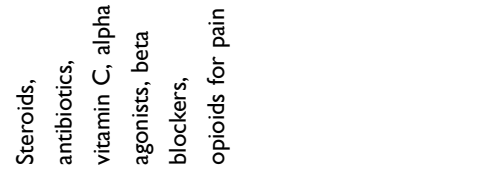 \\
\hline$\Sigma \bar{z}$ & 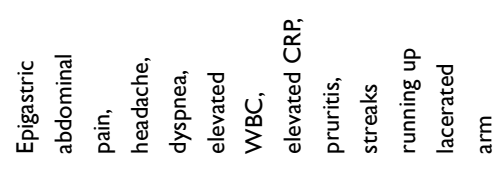 \\
\hline ¿্்ָ & 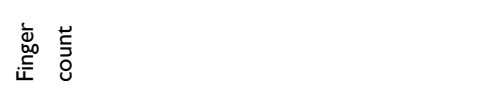 \\
\hline 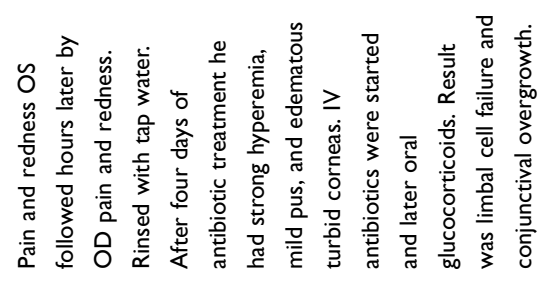 & 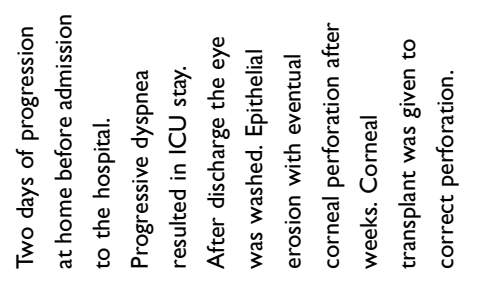 \\
\hline 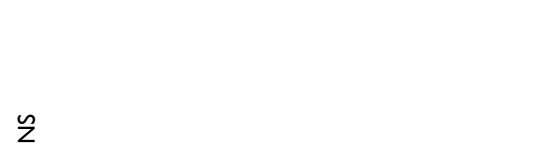 & 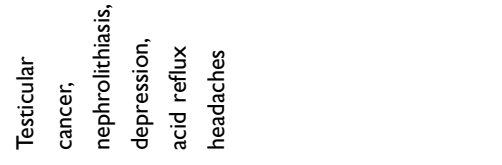 \\
\hline $\begin{array}{l}3 \\
\text { o } \\
\text { ơ } \\
\text { à }\end{array}$ & 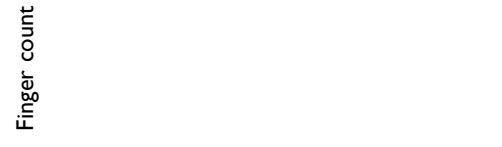 \\
\hline 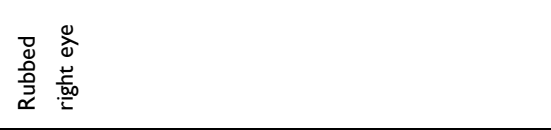 & 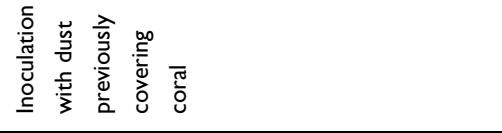 \\
\hline$\sum_{\ddagger}$ & 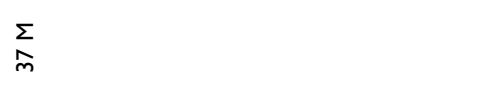 \\
\hline 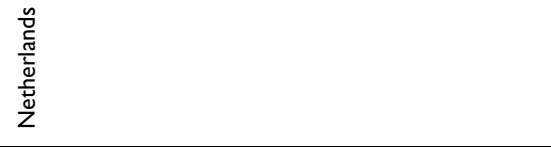 & $\begin{array}{l}\text { 总 } \\
\text { 芯 }\end{array}$ \\
\hline$\stackrel{\circ}{\grave{i}}$ & ఫ్రి \\
\hline$\overline{\bar{o}}$ & $\overline{\bar{o}}$ \\
\hline 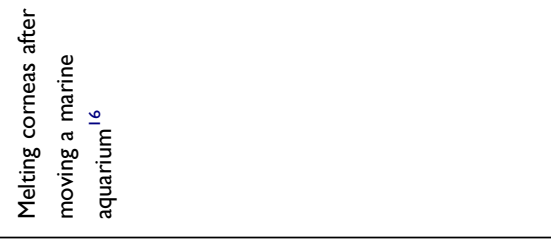 & 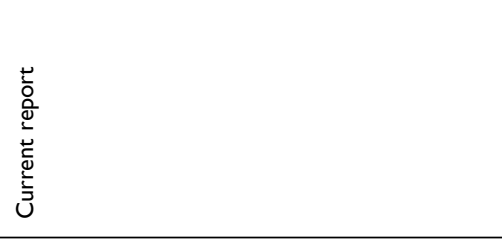 \\
\hline
\end{tabular}


Descemet's membrane were also observed. His WBC had improved (7.3) and broad-spectrum antibiotics were discontinued. He was discharged home (now day four after exposure) on his ophthalmic drops as well as ampicillin and amoxicillin.

The following day (five days after initial exposure), he presented to the clinic for initial evaluation with the author (Hollingshead Eye Center, P.C., Boise, ID) for follow-up where the patient complained of discharge and worsening severe pain in his right eye. His left eye was asymptomatic. He complained of feeling 'sick to his stomach' and had a headache. On examination, his best-corrected visual acuity was to hand motions OD and 20/25 OS. Slit-lamp examination showed upper lid edema and 2+ diffuse conjunctival injection without limbal blanching. Corneal scarring, opacification, and edema were present. Fluorescein (BioGlo Fluorescein Sodium I.P. 1 mg, Omni Lens Pvt. Ltd.) staining revealed $95 \%$ loss of epithelial cells with trace epithelium remaining at the corneal limbus from 10 to 1:30 clock hours. Folds in Descemet's membrane were present and an irregular stroma was observed. Visualization of the anterior structures was difficult due to $2+$ corneal edema, consistent with a Grade III injury in the Hughes classification scheme (as modified by Thoft).

After instilling proparacaine (Proparacaine Hydrochloride Ophthalmic Solution, USP 0.5\%, Alcon Laboratories, Inc), the eye was rinsed with a full bottle of Accu-WASH Eye Irrigating Solution (Accutome) as it had not been previously irrigated. Following the rinse, the patient was again numbed with proparacaine, and a Prokera (BioTissue, Inc) amniotic membrane was placed. Topical prednisolone 1\% (brand unknown) and moxifloxacin $0.5 \%$ (brand unknown) were prescribed four times a day and topical cyclopentolate 1\% (brand unknown) three times a day. A shield at night with shatter-proof glasses and preservative-free artificial tears, one drop hourly while awake, were recommended.

Two days later (seven days after initial exposure), the patient was seen again with a complaint that his amniotic membrane graft had fallen out. The examination was unchanged with the exception that he'd now developed thinning along the inferior limbus. The membrane was replaced, and topical doxycycline $100 \mathrm{mg}$ twice a day with oral vitamin C $1000 \mathrm{mg}$ was added to his treatment regimen. The amniotic membrane fell out again one day later and the decision was made to leave it out since the patient would not tolerate it. On examination four days later (eleven days after exposure), he complained of photophobia and a continued headache. On examination, the opacification had worsened and worsening inferior limbal thinning (now estimated to be 50\% thinned) was noted. The frequency of prednisolone drops was increased to every two hours.

On follow-up one week later, there was no subjective improvement and the eye was still in severe pain. However, there was some improvement, with the limbal epithelial edge healing (epithelium present from 10:00 to $2: 00$ ) but still with an $80 \%$ epithelial defect. The surface layers of the cornea were gelatinous and a bandage contact lens was inserted to help facilitate healing. The inferior thinning remained stable at $50 \%$. Over the course of several more exams in the following two weeks, the patient's pain had decreased significantly, but the photophobia remained. Additional healing was noted at the limbal edge but with no improvement of the thinning along the inferior limbus. The cornea continued to have a gelatinous appearance, with the cornea appearing to shift and move with even gentle pressure such as with the patient blinking.

On day 29 after initial exposure to palytoxin, the patient presented emergently to the clinic with excessive pain and a right-sided headache. He stated that he put some pressure on his eye and eyebrow and it, "gushed water everywhere." Examination showed 2+ Meibomian gland dysfunction OD and possible corneal perforation. Intraocular pressure was unmeasurable. The iris was adherent to the area of presumed perforation; Seidel-sign was negative, and the cornea was so opaque and edematous that the anterior chamber was difficult to visualize. Emergent penetrating keratoplasty was scheduled at the hospital's earliest availability. Brimonidine $0.1 \%$ twice a day was prescribed to minimize aqueous production in the hopes of keeping the iris plugging the presumed corneal perforation until surgery could be done.

On examination the following day, just prior to surgery, 10:00-2:00 epithelialization was stable. Severe thinning along the limbus was noted in the 4:30-7:30 position with a bulging overhanging ledge of swollen white cornea. This thinning was presumed to be the site of perforation. Bullae was present in the inferior cornea. Seidel sign was negative along the entire cornea and limbus.

Surgery consisted of anterior and posterior synechialysis, temporary tarsorrhaphy, and corneal transplant with an $11 \mathrm{~mm}$ corneal graft into an $11 \mathrm{~mm}$ bed - the largest trephines available at the time of surgery. Following uneventful surgery, a successful clear, large corneal scleral transplant with intact sutures was noted. Postoperatively, suture removal was 
completed over the course of 17 months. Mild nonprogressive peripheral anterior synechia was noted in the areas where the graft extended into the sclera, mild posterior synechia, and a $2+$ anterior and posterior subcapsular cataract. Fourteen months postoperatively mild graft rejection was noted and the patient's prednisolone was increased from once daily to every 2 hours for two weeks, then tapered back to once daily. Eighteen months after penetrating keratoplasty the final sutures were removed, the graft remained clear, and the patient's best spectacle-corrected visual acuity was 20/200, pinhole 20/70 (Figure 1). At final follow-up, twenty months following PTX exposure, scleral lens fitting allowed for 20/15 best-corrected visual acuity.

\section{Other Case Reports}

Eighteen accounts of ocular exposure to PTX have previously been reported in the literature. Our case makes nineteen total reported cases of ocular exposure. Seventeen cases have occurred since 2010 (89\%), ${ }^{5,7,11-17}$ with fifteen occurring since $2015(79 \%) .^{7,11-17}$ The only case that was reported outside of North America or Europe occurred in Australia. ${ }^{14}$ Of the eleven cases where the cause of ocular exposure was reported, one case was the result of aquarium water splashing into the eye $(9 \%),{ }^{12}$ in four cases the coral squirted toxin directly into the eye $(36 \%),{ }^{7,13-15}$ two reports resulted from either ocean water exposure ${ }^{18,19}$ or rubbing the eye $\mathrm{e}^{5,16}$ after handling coral (18\% each), one was "exposed to PTX gas" (9\%), and one report resulted from contaminated dust ( $9 \%$ ) though there is a possibility that the patient rubbed their eye and did not recall that

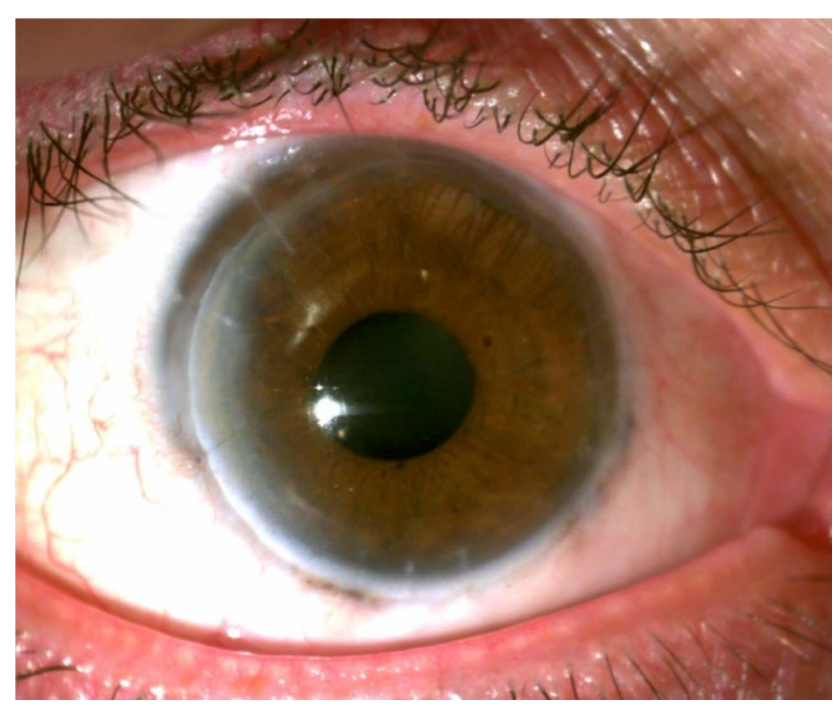

Figure I Right eye of the patient at nearly eighteen months following penetrating keratoplasty. Courtesy of Dr.Ryan T Barrett file photo. during the history. Four of the total cases (21\%) had signs of systemic toxicity. ${ }^{5,7,15}$ All but one case of presumed ocular PTX exposure has led to corneal damage (95\%). ${ }^{14}$

Four cases $(21 \%)$ resulted in corneal perforation. ${ }^{11,17}$ Seven $(39 \%)$ have presented with visual acuity of $20 / 70$ or worse. $^{7,11,16}$ Of the twenty one eyes with reported best correct visual acuity (BCVA) at follow-up, nine (43\%) had $20 / 25$ or better, $5,11,14,19$ eight $(38 \%)$ had BCVA between 20/30 and 20/90, ${ }^{1,12,17}$ with four (19\%) having best-corrected visual acuity of $20 / 100$ or worse at final follow-up. ${ }^{11,16}$ The mildest cases were treated conservatively and had excellent outcomes; treatment was reported in all but one case. Fourteen cases $(73 \%)$ were treated with steroids, ${ }^{5,7,11-19}$ though they were delayed in nine (64\%) of those patients. ${ }^{5,11-14,16,17,19}$ Seventeen patients (89\%) were treated with antibiotics. $5,7,11-19$ Of the twenty-three affected eyes, twelve eyes $(52 \%)$ in nine patients required surgical intervention in the post-exposure period. ${ }^{7,11,12,17}$ In eyes with surgical intervention, the most common procedures were amniotic membrane transplant $(42 \%)^{7,11}$ penetrating keratoplasty $(42 \%),{ }^{11}$ cataract extraction $(17 \%),{ }^{11}$ lateral tarsorrhaphy $(17 \%),{ }^{11}$ and synechialysis $(17 \%) .{ }^{11}$ One eye underwent deep anterior lamellar patch grafting, ${ }^{11}$ and one eye underwent scleral contact lens fitting ( $8 \%$ each). ${ }^{12}$

\section{Discussion}

We present a case of a patient with severe right eye pain following handling of coral which released a cloud of dust that had been covering the coral into the air. After two days of outpatient antibiotic treatment, the patient awoke with eye pain and discharge, difficulty breathing, and systemic symptoms. He was admitted to the hospital and later transferred to the ICU over concerns of respiratory decline. Ophthalmic exam showed 1+ folds in Descemet's membrane and a peripheral inflammatory ring. His bestcorrected visual acuity was to finger count OD. He was treated principally with steroids and antibiotics and was given an amniotic membrane graft. Despite treatment, the patient developed corneal scarring, opacification, and edema and eventual corneal perforation. Penetrating keratoplasty, anterior and posterior synechialysis, temporary tarsorrhaphy, and corneal transplant were used to manage the case. Twenty months after exposure a scleral lens allowed for best-corrected visual acuity of 20/15.

As noted in the case and subsequent literature, ocular exposure to PTX can be a vision-threatening occurrence. These ophthalmic cases are likely underreported and may 
often be subclinical. Patients can be exposed via direct spraying of the toxin into the eye, ${ }^{7,13,14}$ contact with contaminated water, ${ }^{12,18,19}$ or by rubbing the eyes after handling toxin-producing coral. ${ }^{5,11}$ Patient presentation is nonspecific and can include pain, photophobia, a decrease in visual acuity, hyperemia, and a foreign body sensation. ${ }^{5,11,14,17}$ Ocular findings can vary, but most often shows conjunctival injection, a ring-shaped inflammatory infiltrate, and folds in Descemet's membrane. 5,7,11-13,17,19 Iritis, bullae rupture, epithelial defects and corneal erosion and ulceration, white spots on the limbus, limbal stem cell failure, anterior chamber reactions, crystal-like foreign bodies, and conjunctival or punctate tarsal or bulbar hemorrhages may also be present. $7,11-13,15-19$

For patients presenting with presumed ocular PTX exposure, immediate irrigation or instillation of artificial tears should be completed to remove any excess or remaining toxin. Care should be taken not to wash toxin that may be present in one eye into the other eye. ${ }^{7,12}$ The total time of exposure and toxic concentration is possibly increased with contact lens use. Neurotoxic effects of PTX may inhibit corneal nerve transmission, allowing for longer contact time and more destructive consequences. Thus, immediate removal of contact lenses has been recommended. ${ }^{5}$

Subsequent management largely depends on the severity of the PTX contact as assessed clinically. Early aggressive treatment with corticosteroids (eg, 1\% prednisolone acetate at least six times daily), with adjuvant antibiotic prophylaxis, is beneficial in mild cases. ${ }^{11}$ As severity increases, the addition of oral doxycycline, oral steroids, and ascorbic acid should be considered as adjuvant treatment to hourly topical corticosteroid use. ${ }^{11,12}$ Severe exposure may necessitate surgical intervention in addition to medical management to preserve residual visual acuity. As was the case in our patient, corneal transplantation should be considered in cases of corneal perforation. Other published reports indicate that anterior lamellar patch graft may be a viable option. To address concerns of persistent epithelial defects, tarsorrhaphy, epithelialization stimulating eyedrops, or amniotic membrane transplants may be of use. Finally, rigid or scleral contact lenses may improve visual acuity in patients with residual scarring. ${ }^{11,12}$

Although ocular exposure can be vision threatening, the majority of cases, including all known lethal instances, are associated with other routes of PTX contact. While some of these reports have a verified exposure, others are presumed based on the history. ${ }^{10,20}$ We will review case reports published along with their PTX exposure and their clinical course.

Cutaneous exposure to palytoxin is likely the most common route of exposure, since handling the organism can result in epidermal exposure. Similar to ocular exposure, many cases may be subclinical and relatively asymptomatic. Additionally, accidental ocular exposure is likely to present with associated periorbital contact and may present with eyelid swelling. ${ }^{13}$ Cutaneous exposure is believed to result in a contact dermatitis-like reaction on the effected skin. ${ }^{21}$

Online aquarium forums and blogs contain many accounts of potential PTX contact based on symptoms after handling the coral. However, these cases are either subclinical or unreported in the literature; thus, there are limited published case reports on cutaneous exposure. Of the documented reports, patients experienced local erythema, edema, and irritation with more systemic effects such as dizziness, nausea, a metallic taste, perioral numbness, and an urticarial rash..$^{3,8,22,23}$ Interestingly, not all cases of systemic effects are associated with breaks in the epidermal barrier, ${ }^{8}$ indicating that PTX likely can be absorbed through the skin. However, cutaneous absorption is likely minimal as evidenced by the fact that all reported cases were treated supportively and recovery was noted within one week. ${ }^{3,8,22,23}$

To our knowledge, there are fourteen published reports of inhalational PTX exposure comprising forty-four cases. $^{3,9,22,24-33}$ This represents the most reported exposure route in the literature. Of these, nearly all are associated with inhalation of steam or vapor after attempting to clean or remove zoanthid or palythoa from home aquariums with the use of hot or boiling water. The cases occur worldwide, but the majority of reported cases have occurred in the United States. The presenting symptoms are most commonly dyspnea, ${ }^{3,9,22,24,25,27,28,30-32}$ chest pain, ${ }^{3,22,27}$ cough, ${ }^{24,25,}$ $27,28,30,32,34$ tachycardia, $3,9,24,27,28,30,33$ nausea and/or vomiting, $3,27,29,30,33$ headaches, ${ }^{3,30}$ fever, ${ }^{9,24,25,28-33}$ and myalgia or weakness. ${ }^{9,25,26,28,29,31,32}$ Since there is no antitoxin for PTX all patients received symptomatic treatment and all recovered within one month with three exceptions (one patient recovered in three months, one had recurring symptoms after two years, and one had permanent neurologic damage and respiratory symptoms after seven years of follow-up).

Reported cases of foodborne gastrointestinal poisoning are the result of unintentional consumption of various seafood (ie, fish or crab) species contaminated with 
PTX. ${ }^{10,20}$ In these cases, it is presumed that PTX toxicity originated from microalgae that were ingested by bottomfeeding organisms and transmitted through the food chain to larger organisms. Ingestion of PTX has resulted in the only known human fatalities associated with PTX exposure. There are 154 cases well summarized by Tubaro et al with four additional cases later reported by $\mathrm{Wu}$ et al; of the 158 total reports, 138 cases $(87.3 \%)$ had confirmed presence of PTX while 20 were diagnosed on the history and symptomatology, 33 cases $(20.9 \%)$ were ultimately lethal.

Ingestion leads to varied and progressive symptoms that most frequently begin with metallic taste, nausea, vomiting, fatigue, myalgias, diarrhea, extremity and perioral numbness, and black urine, which may progress to organ failure, autonomic instability, delirium. Abdominal pain, convulsions, deafness, electrolyte abnormalities and paralysis were also reported without an associated timeline in some cases. ${ }^{10,20}$ Ultimately, patients may experience hemorrhage, neurologic damage, or ventricular fibrillation which lead to ischemia and death. ${ }^{35}$ Treatment is again primarily supportive, though one patient who was found to be hyperkalemic with associated EKG changes was given IV calcium gluconate and glucose with insulin. Unfortunately, the patient developed asystole and died in the ICU before the initiation of emergent hemodialysis. ${ }^{10}$

Due to the morbidity and mortality associated with palytoxin exposure, avoidance of the toxin should be the primary approach to prevention. Keepers of sea life potentially carrying this toxin should observe precautions when handling these organisms or their environment by using personal protective equipment including eye protection. Additionally, a reporting system to local or national health departments may allow for tracking of sources or origin of corals allowing officials to inform others who may have poisonous marine life. Our findings and other reports of toxic effects reveal the need for caution and regulation in making these f potentially dangerous sea life available to the public.

Palytoxin exposure can affect cells through both direct contact and indirect secondary inflammation. At present, the toxin is known to disrupt ion pumps and the cytoskeleton, in addition to the release of inflammatory cytokines. $^{4,35}$ We discuss each mechanism of action and how it may ultimately impact the cornea.

Cells use ATP-dependent ion channels to move various cations and anions against their concentration gradient and allow for favorable intracellular conditions. Disruption of this process can prove fatal to affected cells. Although the mechanics of the process are not entirely understood, multiple studies have helped elucidate the ability of PTX to bind to the extracellular component of the $\mathrm{Na}+/ \mathrm{K}+$ ATPase pump and transition it from a highly selective energy-dependent pump to a cationic passive ion channel. ${ }^{35}$ This effect results in the accumulation of intracellular calcium and upregulation of downstream signaling cascades, including the MAP kinase pathway. This has been reported to cause neurotransmitter dysregulation as well as neurotoxicity in neuroblastoma cell lines. ${ }^{4}$ These changes have been associated with necrotic changes on cell morphology in multiple cell lines including skin keratinocyte cell lines. $^{35}$

Molecular interactions such as these are the likely cause of corneal epithelial cell death upon PTX exposure. The resulting disruption of the corneal epithelium can result in marginal ulcers and large ring-shaped inflammatory responses as well as swelling of the stromal layer and associated keratitis with penetration into the stroma and endothelium. $^{13}$

Although there is a general understanding of PTX's effects on ion channels, less is known about its interactions with the cytoskeleton. The cytoskeleton regulates many aspects of cellular function, including mechanical support, mitosis, and vesicular transport. Actin, a central component of the cytoskeleton, is a dynamic polymeric structure that constantly undergoes breakdown and remodeling via its monomers. Its function can be interfered with either by binding individual monomers (G-actin) and preventing the formation of microfilament polymers (F-actin) or by binding to the microfilament polymers themselves and initiating depolymerization. ${ }^{36}$

The microfilament aspect of the cytoskeleton is highly regulated by various actin-binding proteins (ABP). These proteins may be affected by dysregulation of ion pumps, which can cause increases in intracellular calcium, a known trigger of apoptosis; and thus, cytoskeleton breakdown and cell death. In recent years, multiple cell lines treated with PTX have shown cytoskeletal decreases in actin as evidenced by laser scanning cytometry. ${ }^{36}$ Treatment with PTX was shown to reduce F-actin levels in multiple cell lines. Interestingly, these decreases in F-actin were also observed in calcium-free medium, giving evidence that PTX can directly affect the microfilament cytoskeleton. All of these findings indicate an intra- and extracellular cytoskeletal destructive process associated with PTX. ${ }^{37}$ These changes in the cytoskeleton may affect the cellular morphology, depending on cell type, ${ }^{36}$ and studies on the 
effects of PTX on corneal cells are needed to understand the effects on this specific cell population better.

One of the most commonly reported signs in ocular exposure is the presence of inflammation, including an inflammatory ring. PTX has been shown to increase the expression of inflammatory cytokines (eg, IL-6, IL-8, TNF-a, $\mathrm{PGE}_{2}$ ), and histamine in human keratinocytes. In addition, these products were found in the growth media indicating an ability for these cells to secrete these upregulators of inflammation. These molecules play a role in neutrophil chemotaxis, pain, swelling, and promoting the inflammatory response. This indicates that the presence of PTX may attract inflammatory cells via a secondary chemotaxic mechanism. ${ }^{21}$

The ionic, inflammatory, and cytoskeletal effects of PTX can result in visually devastating ocular effects. The molecular interactions of the free-flowing ionic pumps likely cause corneal epithelial death soon after exposure. Disruption of the corneal epithelium and release of proinflammatory cytokines can lead to marginal ulcers, a large ring-shaped inflammatory response, and swelling of the stromal layer with associated keratitis. As the toxin burrows through the superficial layers, exposure of subsequent stromal and endothelial tissue can lead to eventual perforation. As the epithelial wound attempts to heal, corneal myofibroblasts express high levels of alphaactin, which may be disrupted by PTX, slowing the body's therapeutic process. ${ }^{12,13}$ Taken together, we propose that the most likely mechanism of significant ocular injury after PTX exposure is as follows: first, epithelial destruction (from pump dysfunction) and inflammatory mediators (from gene expression upregulation) cause inflammatory cell death and infiltration into the cornea. As these superficial cells die, the more permanent stroma is exposed to both the toxin and inflammatory mediators released from adjacent cells and found naturally in the tear film. ${ }^{38}$ At high enough concentrations, proposed direct cytokine and proteolytic activity of PTX results in the degradation of keratocytes, collagen, and proteoglycans, and subsequent corneal thinning, corneal melt, and eventual perforation. ${ }^{5}$

\section{Prognosis}

The prognosis for PTX exposure varies mainly on the exposure route. Ingestion of PTX containing food is the only reported route associated with mortality, though patients were able to recover in a majority of reports. Ocular exposure has the second-highest morbidity; however, prompt aggressive treatment results in a return to baseline acuity in many patients. Severe exposure, however, can lead to lasting visual defects and astigmatism; ${ }^{7}$ limbal stem cell failure with subsequent conjunctival overgrowth and permanent visual loss, ${ }^{16}$ persistent corneal scarring and neovascularization, ${ }^{13}$ and residual bestcorrected visual acuity worse than $20 / 100^{11,16}$ have been reported. Inhalational exposure can result in dire appearing symptomatology (including an ICU stay in our patient), but with appropriate supportive care, nearly all patients make a full recovery. ${ }^{3,9,22,24-32}$ Cutaneous exposure is likely underreported and is the most likely coexisting exposure in ophthalmic patients, though symptoms are short lived and self-resolving. ${ }^{13}$

\section{Conclusion}

We present a case of corneal perforation associated with exposure to toxic coral dust. This report is only the fourth case of corneal perforation following presumed PTX exposure and the first reported case of ocular palytoxin toxicity from a dust-like substance covering the coral in question. The surgical approach to handling these patients is not standardized, and we hope to provide insight into possible treatment options for patients with severe ocular PTX exposure. Additionally, we provide a summary of the mechanism of action, potential concurrent routes of exposure, treatment options, and prognosis for patients who are exposed to PTX. We hope that this allows for quicker recognition and better care for patients who experience this rare toxicity.

\section{Ethics Approval and Informed Consent}

The patient signed an informed consent for the release of clinical information for education and research purposes. This case report was approved by the Hoopes Vision Ethics Board to publish the case details and the patient signed an informed consent to have the case details and any accompanying images published.

\section{Author Contributions}

All authors made a significant contribution to the work reported, whether that is in the conception, study design, execution, acquisition of data, analysis and interpretation, or in all these areas; took part in drafting, revising or critically reviewing the article; gave final approval of the version to be published; have agreed on the journal to which the article has 
been submitted; and agree to be accountable for all aspects of the work.

\section{Funding}

This study was funded by an unrestricted grant from Research to Prevent Blindness (RPB), 360 Lexington Avenue, 22nd Floor New York, NY 10,017. No support was received for the publication of this article.

\section{Disclosure}

None of the authors has any conflict of interest related to this work.

\section{References}

1. Moore RE, Scheuer PJ. Palytoxin: a new marine toxin from a coelenterate. Science. 1971;80-. doi:10.1126/science.172.3982.495.

2. Suh EM, Kishi Y. Synthesis of palytoxin from palytoxin carboxylic acid. J Am Chem Soc. 1994. doi:10.1021/ja00103a065

3. Pelin M, Brovedani V, Sosa S, Tubaro A. Palytoxin-containing aquarium soft corals as an emerging sanitary problem. Mar Drugs. 2016. doi: $10.3390 / \mathrm{md} 14020033$

4. Patocka J, Gupta RC, Wu QH, Kuca K. Toxic potential of palytoxin. J Huazhong Univ Sci Technol - Med Sci. 2015. doi:10.1007/s11596015-1506-3

5. Moshirfar M, Khalifa YM, Espandar L, Mifflin MD. Aquarium coral keratoconjunctivitis. Arch Ophthalmol. 2010. doi:10.1001/ archophthalmol.2010.206

6. Deeds JR, Handy SM, White KD, Reimer JD. Palytoxin found in Palythoa sp. zoanthids (Anthozoa, Hexacorallia) sold in the home aquarium trade. PLoS One. 2011. doi:10.1371/journal.pone.0018235

7. Ruiz Y, Fuchs J, Beuschel R, Tschopp M, Goldblum D. Dangerous reef aquaristics: palytoxin of a brown encrusting anemone causes toxic corneal reactions. Toxicon. 2015. doi:10.1016/j. toxicon.2015.09.001

8. Nordt SP, Wu J, Zahller S, Clark RF, Cantrell FL. Palytoxin poisoning after dermal contact with zoanthid coral. J Emerg Med. 2011. doi:10.1016/j.jemermed.2009.05.004

9. Hall C, Levy D, Sattler SA. Case of palytoxin poisoning in a home aquarium enthusiast and his family. Case Rep Emerg Med. 2015. doi: $10.1155 / 2015 / 621815$

10. Wu ML, Yang CC, Deng JF, Wang KY. Hyperkalemia, hyperphosphatemia, acute kidney injury, and fatal dysrhythmias after consumption of palytoxin-contaminated Goldspot herring. Ann Emerg Med. 2014. doi:10.1016/j.annemergmed.2014.06.001

11. Farooq AV, Gibbons AG, Council MD, et al. Corneal toxicity associated with aquarium coral palytoxin. Am J Ophthalmol. 2017. doi:10.1016/j.ajo.2016.10.007

12. Barbany M, Rossell M, Salvador A. Toxic corneal reaction due to exposure to palytoxin. Arch Soc Esp Oftalmol. 2019. doi:10.1016/j. oftal.2018.10.011

13. Chaudhry NL, Przybek J, Hamilton A, Carley F. Unique case of palytoxin-related keratitis. Clin Exp Ophthalmol. 2016. doi:10.1111/ ceo. 12768

14. Cheung KG, Haynes R, Porter A, O'Hagen S. Palytoxin exposure causing prolonged conjunctivitis and episcleritis without corneal involvement. J Ophthalmol Vis Neurosci. 2017;2(2).

15. Gaudchau A, Pfeiffer N, Gericke A. Chemical burns caused by crust anemone. Ophthalmologe. 2019. doi:10.1007/s00347-018-0742-9

16. Jalink MB, van Luijk CM. Wegsmeltende hoornvliezen na de verhuizing van een zeeaquarium. Ned Tijdschr Geneeskd. 2019.
17. Wonneberger W, Claesson M, Zetterberg M. Corneal perforation due to palytoxin exposure of domestic zoanthid corals. Acta Ophthalmol. 2020. doi:10.1111/aos.14594

18. Steel DHW. "Dead man's finger" keratoconjunctivitis [2]. $\mathrm{Br}$ J Ophthalmol. 1993. doi:10.1136/bjo.77.1.63-a

19. Keamy J, Umlas J, Lee Y. Red coral keratitis. Cornea. 2000. doi:10.1097/00003226-200011000-00021

20. Tubaro A, Durando P, Del Favero G, et al. Case definitions for human poisonings postulated to palytoxins exposure. Toxicon. 2011. doi:10.1016/j.toxicon.2011.01.005

21. Pelin M, Florio C, Ponti C, et al. Pro-inflammatory effects of palytoxin: an: in vitro study on human keratinocytes and inflammatory cells. Toxicol Res (Camb). 2016. doi:10.1039/c6tx00084c

22. Deeds JR, Schwartz MD. Human risk associated with palytoxin exposure. Toxicon. 2010. doi:10.1016/j.toxicon.2009.05.035

23. Hoffmann K, Hermanns-Clausen M, Buhl C, et al. A case of palytoxin poisoning due to contact with zoanthid corals through a skin injury. Toxicon. 2008. doi:10.1016/j.toxicon.2008.03.009

24. Tartaglione L, Pelin M, Morpurgo M, et al. An aquarium hobbyist poisoning: identification of new palytoxins in Palythoa cf. toxica and complete detoxification of the aquarium water by activated carbon. Toxicon. 2016. doi:10.1016/j.toxicon.2016.08.012

25. Thakur LK, Jha KK. Palytoxin-induced acute respiratory failure. Respir Med Case Reports. 2017. doi:10.1016/j.rmcr.2016.10.014

26. Schulz M, Łoś A, Szabelak A, Strachecka A. Inhalation poisoning with palytoxin from aquarium coral: case description and safety advice. Arh Hig Rada Toksikol. 2019. doi:10.2478/aiht-2019-70-3209

27. Wieringa A, Bertholee D, Ter Horst P, Van Den Brand I, Haringman J, Ciminiello P. Respiratory impairment in four patients associated with exposure to palytoxin containing coral. Clin Toxicol. 2014. doi:10.3109/15563650.2013.878867

28. Hamade AK, Deglin SE, McLaughlin JB, Deeds JR, Handy SM, Knolhoff AM. Suspected palytoxin inhalation exposures associated with zoanthid corals in aquarium shops and homes - alaska, 2012-2014. Morb Mortal Wkly Rep. 2015. doi:10.15585/mmwr. mm6431a4

29. Snoeks L, Veenstra J. A family with fever after cleaning a sea aquarium. Ned Tijdschr Geneeskd. 2012.

30. Bernasconi M, Berger D, Tamm M. Aquarism: an innocent leisure activity? Respiration. 2012. doi:10.1159/000338789

31. Sud P, Su MK, Greller HA, Majlesi N. Case series: inhaled coral vapor-toxicity in a tank. $J$ Med Toxicol. 2013. doi:10.1007/s13181013-0307-x

32. Rumore MM, Houst BM. Palytoxin poisoning via inhalation in pediatric siblings. Int $J$ Case Reports Images. 2014. doi:10.5348/ ijcri-201488-cr-10399

33. Chang E, Deeds J, Spaeth K. A case of long-term neurological and respiratory sequelae of inhalational exposure to palytoxin. Toxicon. 2020. doi:10.1016/j.toxicon.2020.07.018

34. Cohen AK, Theotoka D, Galor A. Epipremnum aureum keratopathy: case report and review of the literature. Eye Contact Lens Sci Clin Pract. 2019. doi:10.1097/ICL.0000000000000675

35. Patocka J, Nepovimova E, Wu Q, Kuca K. Palytoxin congeners. Arch Toxicol. 2018. doi:10.1007/s00204-017-2105-8

36. Louzao MC, Ares IR, Cagide E, et al. Palytoxins and cytoskeleton: an overview. Toxicon. 2011. doi:10.1016/j.toxicon.2010.09.017

37. Ares IR, Louzao MC, Vieytes MR, Yasumoto T, Botana LM. Actin cytoskeleton of rabbit intestinal cells is a target for potent marine phycotoxins. J Exp Biol. 2005. doi:10.1242/jeb.01897

38. Yüksel Elgin C, İskeleli G, Talaz S, Akyol S. Comparative analysis of tear film levels of inflammatory mediators in contact lens users. Curr Eye Res. 2016. doi:10.3109/02713683.2015.1037001 


\section{Publish your work in this journal}

Clinical Ophthalmology is an international, peer-reviewed journal covering all subspecialties within ophthalmology. Key topics include: Optometry; Visual science; Pharmacology and drug therapy in eye diseases; Basic Sciences; Primary and Secondary eye care; Patient Safety and Quality of Care Improvements. This journal is indexed on PubMed
Central and CAS, and is the official journal of The Society of Clinical Ophthalmology (SCO). The manuscript management system is completely online and includes a very quick and fair peer-review system, which is all easy to use. Visit http://www.dovepress.com/ testimonials.php to read real quotes from published authors. 